\title{
Fatigue life estimation under multi-axial random loading in light poles
}

\author{
Kazem Reza Kashyzadeh ${ }^{1}$ \\ ${ }^{1}$ Young Researchers and Elite Club, Semnan Branch, Islamic Azad University, Semnan, Iran
}

\begin{abstract}
In the present paper, fatigue life of light poles under multi-axial random loading based on the variable wind direction and speed has been studied. To achieve these purposes, light pole is simulated with all loading conditions in ABAQUS Software. To consider to the three storm days as the critical loading conditions, Random vibration analysis has been performed to obtain stress histories and equivalent stress. Finally, Calculate fatigue life of light pole by using Dirlik Theory.
\end{abstract}

Keywords: Fatigue life, light poles, wind loading, multi-axial loading, Dirlik theory. 


\section{Introduction}

The impact of a lighting system for the comfort and convenience of night driving on city streets is obvious, but the effect on outer-city roads and highways is more directly related to the amount of night driving accidents and often harmful and irreparable damage to the driver and others will enter.

Among the issues that the electric lights and other similar structures, such as lighting masts, antenna masts and must be reviewed and considered..... In addition to the basic size and brightness, followed by the arm and illumination, Structural vibrations caused by wind speed and wind direction. For this purpose it is necessary to apply a combination of the dynamic behaviour of structures under multiaxial random loading involve of speed and wind direction are examined.

During the light structure, underneath the base of a rectangular notch is caused to pass through electrical cables which often have sharp edges and in some cases that experienced people are used to install, Chamfer sharp corners are cut off and the curved shape.

After a period of time, breaks the structure caused by fatigue crack growth under wind loading include in 2 influence parameters such as speed and wind direction. Since fatigue failure occurs suddenly and without any warning, Very dangerous and should be studied on this phenomenon. So, a large number of researchers have investigated it from different points:

Astam et al. examined the fatigue life of steel plates with power poles (Poles, traffic lights and driving) for road construction [1].

Connor et al. studied the fatigue crack growth the inspection of the upper mast lighting towers began. In this way the laboratory deep studies on the dynamic behaviour and high mast lighting structures are discussed in relation to fatigue failures, So much attention on the thickness of steel based plate and the stress distribution in welded joint on the wall of the building [2].

Rode, completed his master's thesis in 2010 as Fatigue performance of rice in light beams. In this thesis, flexural tests on half of Structures Department of Transportation has been done by the Copper Development Association. He is determined stress concentration factor at the notch by using strain measurements. Finally, a new scheme based on a previous design was presented with minor changes where the outer radius of notch increases and then re-test on a sample of the new plan, increase fatigue life of structures has been reported [3].

Manis et al. studied the vibrations caused by the wind on structures of lighting beams.

In this study, all the lights are swinging in the wind power wind speeds Between 17 to $28 \mathrm{mph}$ with a top speed of $48 \mathrm{mph}$ and a storm were examined during which the beam moving at about 8 to 12 inches have been reported.

Following the failure of the welded joint are some beams Check out the main reason growth of fatigue cracks in structures have been reported [4].

Some of the multi-axial fatigue criteria have been compared and reported advantages and limitations for them [5]. In another research, performed tests to obtain fatigue life of components under multi-axial random loading and compared with four published theories. Finally, reported that life prediction based on Dirlik Theory is very close to the experimental results [6].

\section{Finite Element Simulation}

\subsection{Geometric Modelling}

In this study, the structure consists of two parts include in:

\section{Basis of pole}

2. The arm of the light beam is placed in the area.

At the first level, according to the specifications received from the Department of Transportation (Table.1) modelled both part of the structural and then the next step at the base and at a distance of half a meter above the ground exactly in line with the overhead lighting, a rectangular notch (to pass through the wire cable) is created. 
Table1: The characteristics of geometric model [7]

\begin{tabular}{|l|l|l|}
\hline Components & Basis of Pole & Arm of Light \\
\hline Length $(\mathrm{m})$ & 7 & 1 \\
\hline Diameter $(\mathrm{mm})$ & 89 & 28 \\
\hline Thickness $(\mathrm{mm})$ & 3.6 & 2.1 \\
\hline Angle (degrees) & 90 & 30 \\
\hline
\end{tabular}

In order to find finite element simulation of the base and arm lighting poles, the linear elements with square features three-dimensional stress is used. So, can view the notch shot that networking is also seen in Figure.1.

The elasticity theories can be accurately respond well and plasticity theories are neglected. On the other hand, the study of high-cycle fatigue life of the system, does not enter the equation associated with plastic deformation and elastic theory can be used as well.

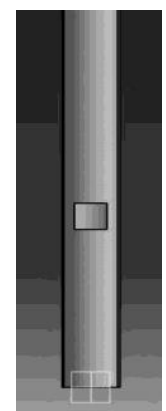

Figure1: Structural FE model with square-shaped opening at the height of half a meter from the ground

\subsection{Materials Information}

In the present work, the piece made of AISI-8620 that is a ferrite-pearlite metal. So, mechanical properties can be seen in Table 2:

Table2: Mechanical properties of ferrite-pearlite metal (AISI-8620)

\begin{tabular}{|l|l|l|}
\hline Properties & Metric & Imperial \\
\hline Density & $7.85 \mathrm{gr} / \mathrm{cm} 3$ & $0.284 \mathrm{lb} / \mathrm{in} 3$ \\
\hline Tensile Strength & $530 \mathrm{Mpa}$ & $76900 \mathrm{psi}$ \\
\hline Yeild Strength & $385 \mathrm{Mpa}$ & $55800 \mathrm{psi}$ \\
\hline Elastic Modulus & $\begin{array}{l}190- \\
210 \mathrm{Gpa}\end{array}$ & $\begin{array}{l}27557- \\
30458 \mathrm{ksi}\end{array}$ \\
\hline Bulk Modulus & $140 \mathrm{Gpa}$ & $20300 \mathrm{ksi}$ \\
\hline Shear Modulus & $80 \mathrm{Gpa}$ & $11600 \mathrm{ksi}$ \\
\hline Poisson's Ratio & $0.27-0.30$ & $0.27-0.30$ \\
\hline $\begin{array}{l}\text { Hardness } \\
\text { Rockwell B }\end{array}$ & 80 & 80 \\
\hline
\end{tabular}

\subsection{Loading}

History load includes time information about weather with two parameters (wind direction and wind speed) of Semnan city based on original data from the Bureau of Meteorology has developed and available for 3 days with the worst (almost stormy weather) that wind direction and velocity versus time as shown in Figure 2 and Figure 3 respectively.

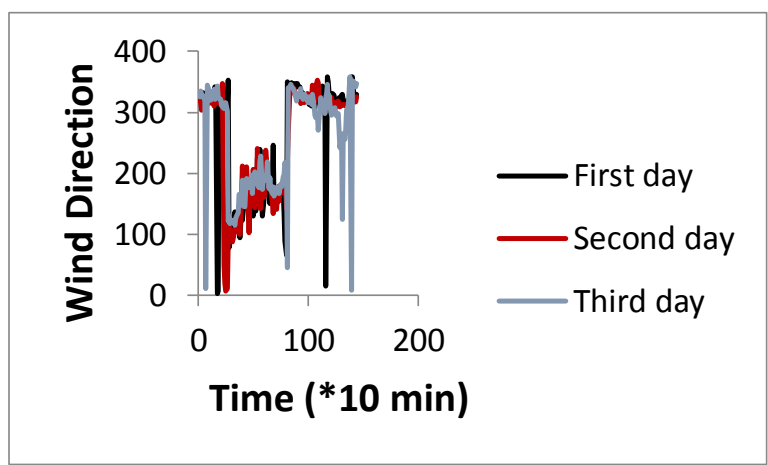

Figure2: wind direction in terms of time during 3 days [8]

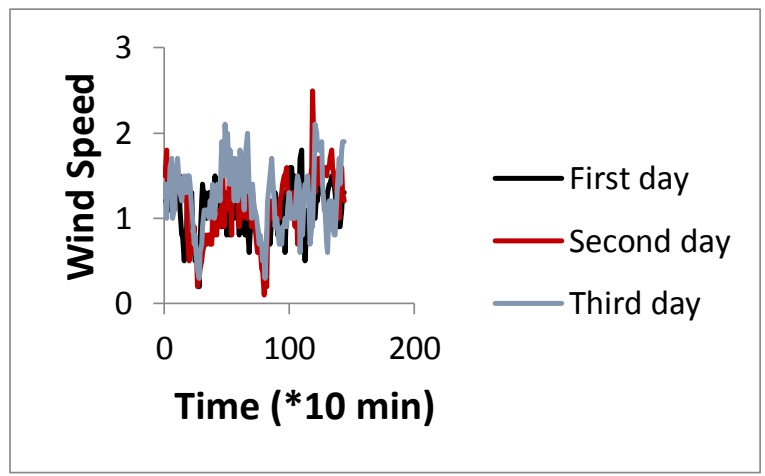

Figure3: wind speed in terms of time during 3 days [8]

It is important that the reported values for determining wind direction is based on Figure.4.

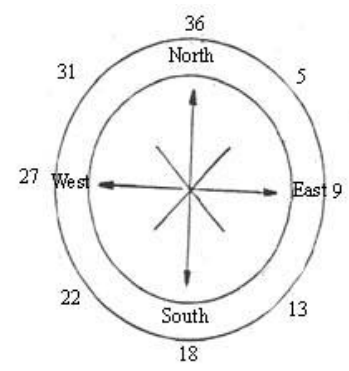

Figure4: Standard for definition for wind direction [8] 


\section{Stress estimation on the structure}

Different standards with formulas are presented to calculate the pressure caused by wind on structures. So, can obtain applied stress on the structure by regarding the cross section of loading.

In this study, used method of Generic Form that neglected temperature effects.

$$
\begin{aligned}
& F=A \times P \times C d \rightarrow \frac{F}{A}=P \times C d \rightarrow \sigma=P \times C d \\
& P=.00256 \times V^{2}
\end{aligned}
$$

That Cd is Drag Coefficient of wind. For long cylindrical shapes such as tubes antenna and beam brightness has been reported equal 1.2 [9].

By substituting speed wind and other data to the above formula, we have:

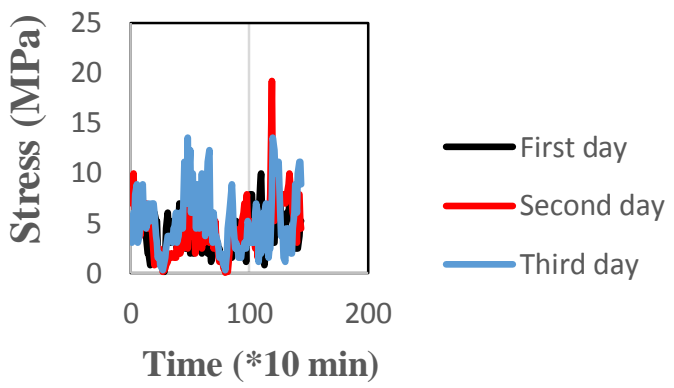

Figure5: applied stress on the light pole caused by speed wind

If the cross section of light pole to be considered as a circle. So, have 4 vertical planes in the range of angle is 90 degree that each plane contains 3 random loadings such as: $L_{1}(t), L_{2}(t), L_{3}(t)$

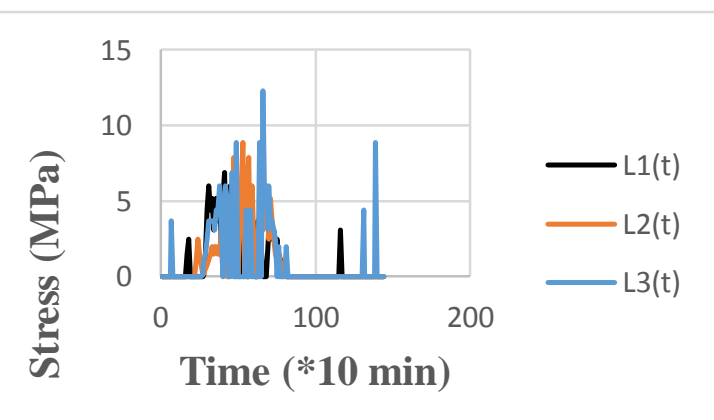

Figure6: stress on the first plane of light pole by caused wind speed

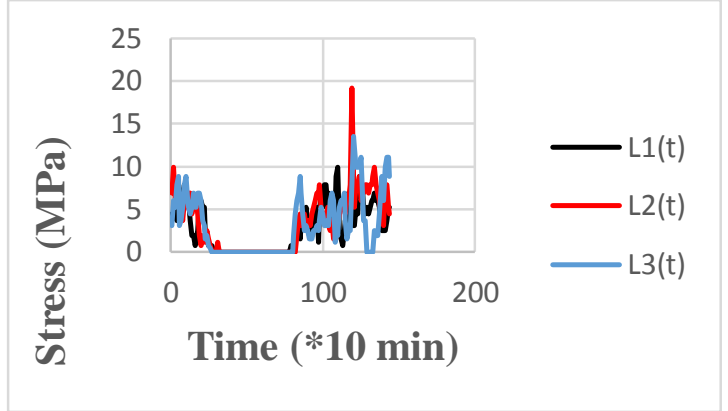

Figure7: stress on the second plane of light pole by caused wind speed

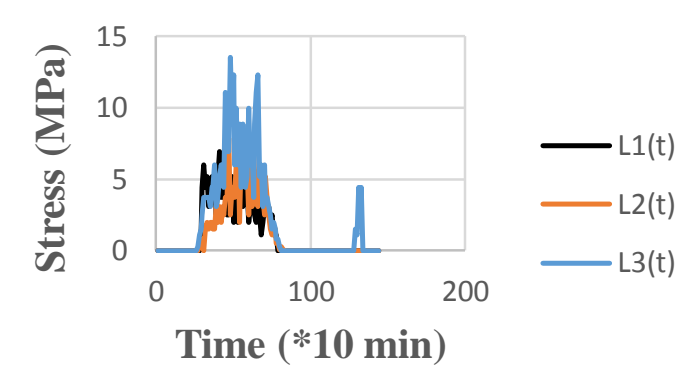

Figure8: stress on the third plane of light pole by caused wind speed

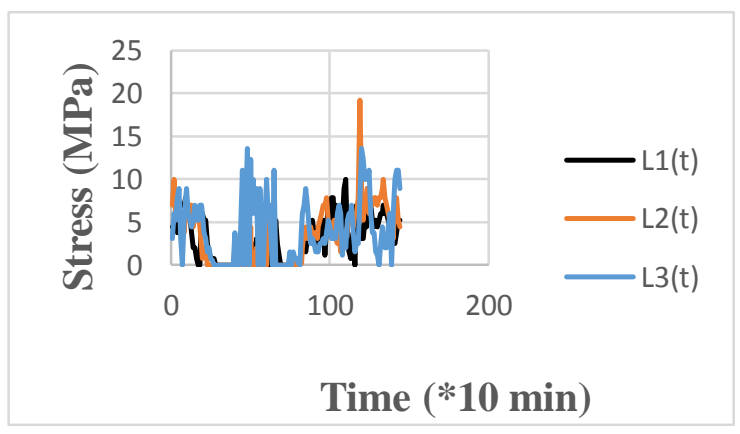

Figure9: stress on the forth plane of light pole by caused wind speed

\section{Fatigue life estimation}

Linear elastic analysis is done under multi-axial loading on the three days. So, strain histories in the different directions have been extracted from ABAQUS Software. 


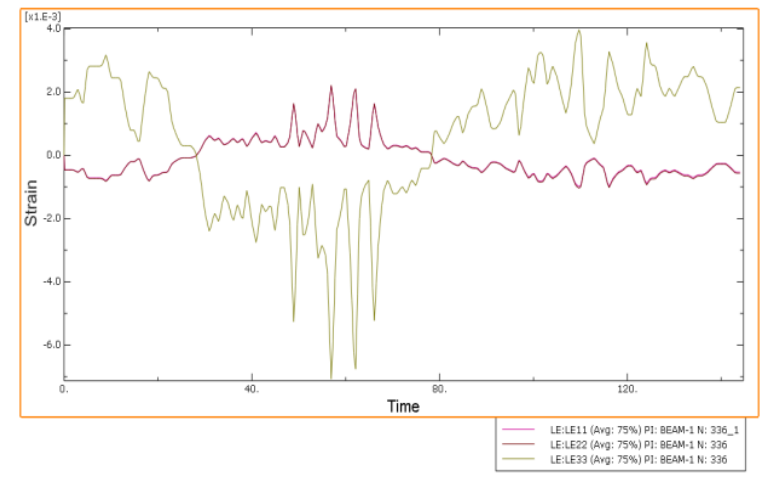

Figure10: strain histories in the different directions on the light pole for the first day

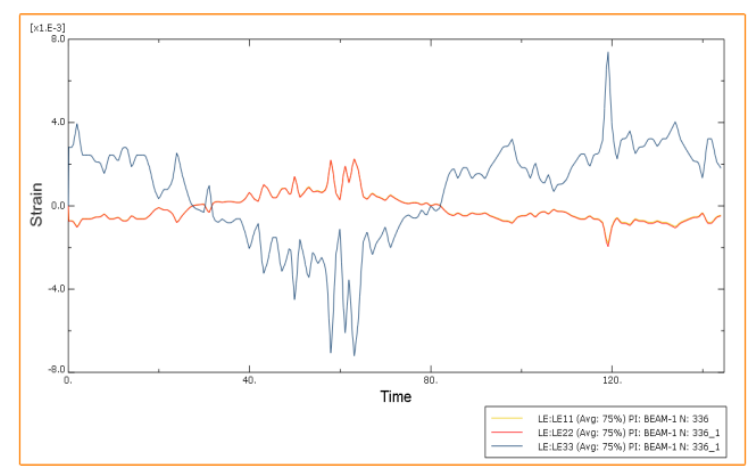

Figure11: strain histories in the different directions on the light pole for the second day

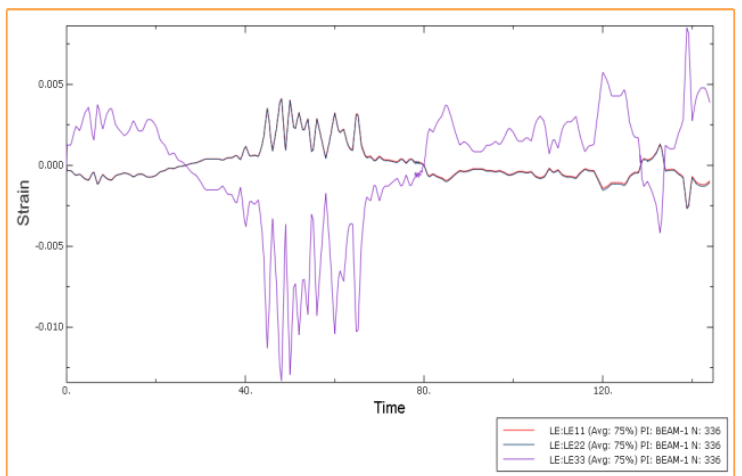

Figure12: strain histories in the different directions on the light pole for the third day

Matrix of transition coefficients can be obtained such as $Q$ between the load and the components of strain tensor.

$$
\begin{aligned}
& L(t)=\left[L_{1}(t), L_{2}(t), L_{3}(t)\right] \\
& \varepsilon(t)=L(t) Q
\end{aligned}
$$

So that:

$$
\begin{gathered}
\varepsilon(t)= \\
{\left[\varepsilon_{x x}(t), \varepsilon_{y y}(t), \varepsilon_{z z}(t), \varepsilon_{x y}(t), \varepsilon_{x z}(t), \varepsilon_{y z}(t)\right]}
\end{gathered}
$$

\begin{tabular}{|c|c|c|c|c|c|}
\hline & 231. & 51. & 78.73 & 77.82 & 85 \\
\hline & so & 203. & 78 & 77.78 & 85.1 \\
\hline & 203.07 & 201.36 & 80.5 & 77.89 & 9.35] \\
\hline
\end{tabular}

Transfer matrix will be:

$$
Q=\left[\begin{array}{ll}
Q_{x x}, Q_{y y}, Q_{z z}, Q_{x y}, Q_{x z}, Q_{y z} & \text { for } L_{1}(t) \\
Q_{x x}, Q_{y y}, Q_{z z}, Q_{x y}, Q_{x z}, Q_{y z} & \text { for } L_{2}(t) \\
Q_{x x}, Q_{y y}, Q_{z z}, Q_{x y}, Q_{x z}, Q_{y z} & \text { for } L_{3}(t)
\end{array}\right]
$$

And has been obtained for the present problem:

By assuming normal distribution (Gaussian) for the probability function of loading, it is enough to follow three below steps to calculate fatigue life of light pole [10].

\subsection{First Step}

Determine the spectral density function directly from the matrix of spectral density loading. The matrix of normalized coefficient of Hooke's Law is made as follows:

$$
G_{\sigma}(f)=\left[Q Q_{H}\right]^{T} G_{L}(f) Q Q_{H}
$$

$\left.\begin{array}{cccccc}Q_{H}= & & & & & \\ 0.2393 & 0.0954 & 0.0954 & 0 & 0 & 0 \\ 0.0954 & 0.2393 & 0.0954 & 0 & 0 & 0 \\ 0.0954 & 0.094 & 0.2393 & 0 & 0 & 0 \\ 0 & 0 & 0 & 0.1439 & 0 & 0 \\ 0 & 0 & 0 & 0 & 0.1439 & 0 \\ 0 & 0 & 0 & 0 & 0 & 0.1439\end{array}\right]$

\subsection{Second Step}

Calculate the spectral density of the time history of stress. So, it is necessary to select right and proper multi-axial fatigue criteria. Thus, used a linear multi-axial fatigue criteria that:

$$
G_{\sigma_{e q}}(f)=a G_{\sigma}(f) a^{T}
$$

In the next level, the maximum normal stress criterion is used on the critical plane.

$$
\begin{gathered}
\sigma_{e q}(t)=l_{\eta}^{2} \sigma_{x x}(t)+m_{\eta}^{2} \sigma_{y y}(t)+n_{\eta}^{2} \sigma_{z z}(t)+ \\
2 l_{\eta} m_{\eta} \sigma_{x y}(t)+2 l_{\eta} n_{\eta} \sigma_{x z}(t)+2 m_{\eta} n_{\eta} \sigma_{y z}(t)
\end{gathered}
$$

Where $l_{\eta}, m_{\eta}, n_{\eta}$ are directional values of normal vector of cosine in related to critical plane.

$$
a=\left[l_{\eta}^{2}, m_{\eta}^{2}, n_{\eta}^{2}, 2 l_{\eta} m_{\eta}, 2 l_{\eta} n_{\eta}, 2 m_{\eta} n_{\eta}\right]
$$

And the critical plane has the maximum variance on the normal stress. 


$$
\mu_{\text {max }}=\max \left(a_{i} \int_{0}^{\infty} G_{\sigma}(f) d f a_{i}^{T}\right)
$$

So, equivalent Von-Misses stress was obtained on the critical point (top right corner of notch) of the structure for each level of loading.

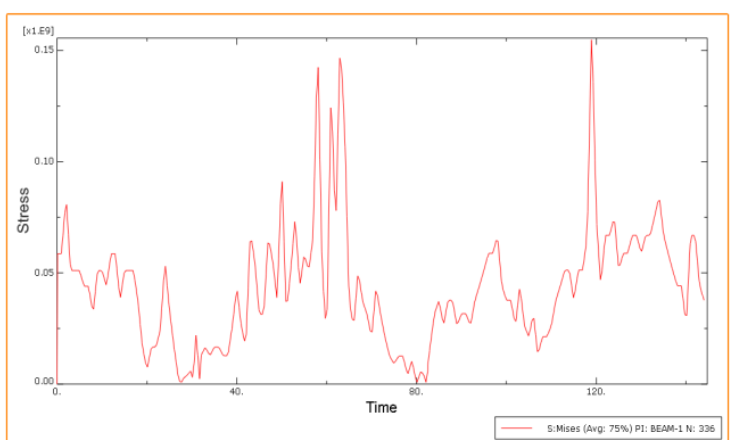

Figure17: equivalent Von-Misses stress for the first day

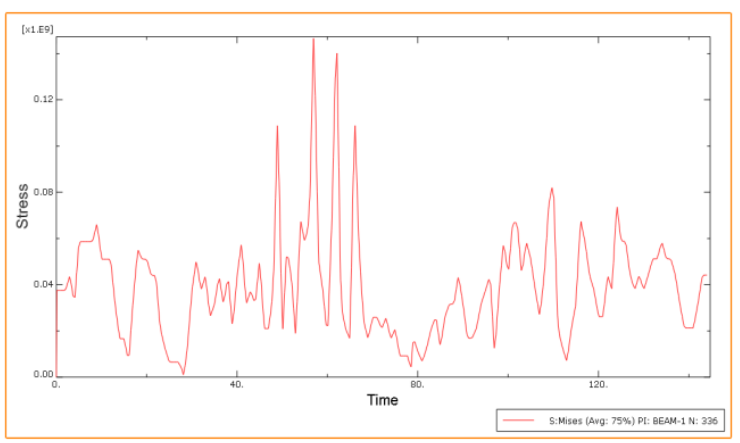

Figure18: equivalent Von-Misses stress for the second day

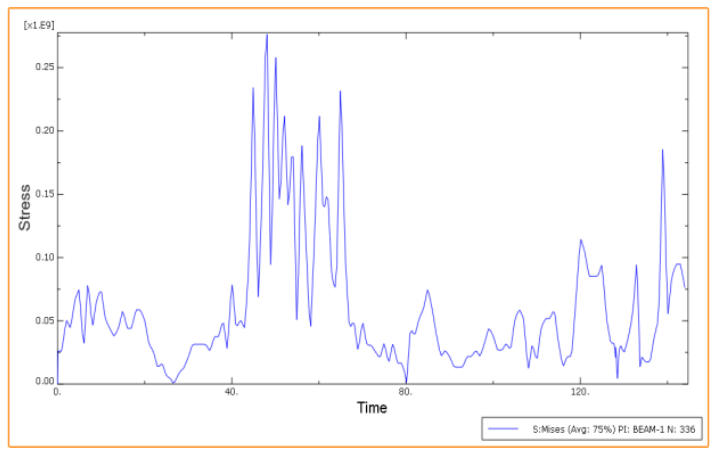

Figure19: equivalent Von-Misses stress for the third day

Finally, calculate power spectral density (PSD) of equivalent stress by using Fast Fourier Transform (FFT).

\subsection{Third Step}

In the present step, is used Dirlik Method to estimate fatigue life of light pole [11-12].

$$
P(\Delta \sigma)=\frac{1}{2 \sqrt{\xi_{0}}}\left(\frac{k_{1}}{k_{4}} e^{\frac{-z}{k_{4}}}+\frac{k_{2} z}{R^{2}} e^{\frac{-z^{2}}{2 R^{2}}}+K_{3} z e^{\frac{-z^{2}}{2}}\right)
$$

$Z=\frac{\Delta \sigma}{2 \sqrt{\xi_{0}}}$

$k_{1}=\frac{2\left(x_{m}-I^{2}\right)}{1+I^{2}}$

$k_{2}=\frac{1-I-k_{1}+k_{1}^{2}}{1-R}$

$k_{3}=1-k_{1}-k_{2}$

$k_{4}=\frac{5\left(I-k_{3}+k_{2} R\right)}{4 k_{1}}$

$R=\frac{1-x_{m}-k_{1}^{2}}{1-I-k_{1}-k_{1}^{2}}$

$x_{m}=\frac{\xi_{1}}{\xi_{0}} \sqrt{\frac{\xi_{2}}{\xi_{4}}}$

$I=\frac{\xi_{2}}{\sqrt{\xi_{0} \xi_{4}}}$

$\xi_{k}=\int_{0}^{\infty} G_{\sigma_{e q}}(f) f^{k} d f$

The fatigue damage accumulation was performed with the use of the linear PalmgrenMiner hypothesis for the whole stress range [11]:

$$
T_{c a l}=\left(M^{+} \int_{0}^{\infty} \frac{P\left(\Delta \sigma_{t r}\right)}{N\left(\Delta \sigma_{t r}\right)} d \Delta \sigma_{t r}\right)^{-1}
$$

Where [11-12]:

$$
\begin{aligned}
& M^{+}=\sqrt{\frac{\xi_{4}}{\xi_{2}}} \\
& N\left(\Delta \sigma_{t r}\right)=\sigma_{a f}^{m} N_{0}\left(\frac{\Delta \sigma_{t r}}{2}\right)^{-m}
\end{aligned}
$$

\section{Conclusion}

In this study, fatigue life of light poles under worst weather conditions have been obtained infinite years to compare with material properties and without crack and other defects. On the other hand, fatigue life of structure is more than 30 years old. This value is calculated based on the load history as variable amplitude functions and are neglected temperature changes in during day. Therefore, it is suggested that Temperature changes should be considered in future research and will do a coupling of thermal and structural analysis.

However, if there is a crack on the one corner of notch caused by manufacture level, fatigue life of 
structure is not infinite and should be calculated by using crack growth and failure criteria.

\section{References}

A. Stam, N. Richman, C. Pool, C. Rios, T. Anderson, K. Frank, (2011). Fatigue Life of Steel Base Plate to Pole Connections for Traffic Structures. Center for Transportation Research. The University of Texas at Austin

J. Conner, C. Hodgson, Fatigue Cracking and Inspection of High-mast Lighting Towers. Iowa DOT, IBC-05-31

J.T. Rudd, (2010). Fatigue Performance of Brass Light Pole Couplings. Thesis for the degree of Master of Science in Civil Engineering, University of Illinois at Urbana-Champaign

P. Manis, P.E. Wes Jones, (2008). Wind induced vibrations on light standards. structure magazine

Adam Nieslony, (2010). Comparison of some selected multiaxial fatigue failure criteria dedicated for spectral method. Journal of Theoretical and Applied Mechanics

Adam Nieslony, (2012). Fatigue Life prediction for broad-band multiaxial loading with various PSD. International Journal of Fatigue

Characteristics of geometry model of light poles, (2013). Department of Transportation, Semnan city

Data on the wind. (2013). Meteorological Office, Semnan city

D. B. Leeson, (1992). Book of Physical Design of Yagi Antennas. American Radio Relay League

A. Nielsony, E. Macha, (2007). Spectral Method in Multiaxial Random Fatigue. Lecture notes in applied and computational mechanics, springer

M. Mrsnik, J. Slavic, M. Boltezar, (2013). Frequency-domain methods for a vibration fatigue life estimation - application to real data. International Journal of Fatigue, Vol. 47

T. Irvine, (2014). Experimental Verification of the Dirlik Fatigue Cycle Method 